\title{
Continuum probe two-dimensional electronic spectroscopy of the photosystem II reaction center
}

\author{
F. D. Fuller, J. P. Ogilvie \\ Department of Physics and Biophysics, University of Michigan, Ann Arbor, Michigan 48109
}

\begin{abstract}
We report two-dimensional electronic spectroscopy of the photosystem II reaction center, collected in the pump-probe geometry employing a continuum probe. This enables observation of ion bands that report on intermediates in the charge separation process.
\end{abstract}

Two-dimensional electronic spectroscopy (2DES) has emerged as an attractive method for observing femtosecond and picosecond dynamics of a broad range of condensed matter systems [1]. As a third order spectroscopy, 2DES contains similar information to more standard transient absorption techniques, with the distinct advantage, among others, that it supplies excitation dependence of the transient signal without sacrificing temporal resolution. Following work in the infrared [2], we previously demonstrated that a transient absorption setup with broadband probing can be easily adapted to perform 2DES through the insertion of a pulse shaper in the pump beam [3]. We present here some additional improvements to our prior method and demonstrate their application to the D1D2-cyt-b559 photosystem II reaction center (PSII RC) purified from spinach.

The photosystem II complex (PSII) is unique among biological systems in its ability to harness solar energy to split water [4]. A desire to understand the fundamental structure-function relationship, as well as the potential to create artificial mimics has inspired a wealth of studies aimed at understanding PSII's unique capabilities. PSII is composed of over 25 subunits that bind $>250$ chlorophyll molecules acting as light harvesting antennae to transfer energy to the PSII reaction center (RC). The primary processes in photosystem II are the absorption of light energy by the lightharvesting antennae and the transfer of energy to the photosystem II reaction center (PSII RC). The PSII RC is composed of the D1 and D2 proteins that bind the cofactors necessary for solar energy conversion. Simplified preparations containing the D1 and D2 proteins as well as cytochrome b559, but lacking the antennae proteins and reducing side plastoquinone acceptors are frequently used to study of the processes of energy and charge transfer within the PSII RC. There have been a wealth of spectroscopic studies of the PSII RC [5, 6]. Despite this intense research effort, the mechanisms and timescales of the ultrafast energy transfer and charge separation processes are not yet fully understood [7]. Determining the charge separation pathways and testing excitonic models [8] of the PSII RC motivate this research. Assigning transient spectral features observed to specific energy transfer and charge separation processes can be challenging, however, as the excitons are closely spaced in energy and their linewidths all overlap, even at low temperature. Further complicating matters, the spectroscopic signatures of energy transfer and charge separation are difficult to distinguish, and share similar timescales. To tackle the issue of spectral overlap, collecting the data under different conditions may be used in conjuction with a global model to tease apart the kinetics. Previous pump probe studies have aimed to deconvolute the kinetics of overlapping features by

This is an Open Access article distributed under the terms of the Creative Commons Attribution License 2.0, which permits unrestricted use, distribution, and reproduction in any medium, provided the original work is properly cited. 
selectively exciting across the $\mathrm{Q}_{\mathrm{y}}$ band (660-690 $\mathrm{nm}$ ) with a narrowband pump [9]. In these studies a broadband probe enabled the indentification of charge separation intermediates, leading to the proposal that two distinct charge separation pathways are active at $77 \mathrm{~K}$. We have previously performed 2DES on the $\mathrm{Q}_{\mathrm{y}}$ band of the PSII RC [10], directly observing excitonic coupling in the system, and mapping the exchange of energy among excitonic states with high time resolution. In this study we extend the spectral window of our previous measurements, collecting 2DES of the PSII $\mathrm{RC}$ with a continuum probe spanning 450-700 $\mathrm{nm}$. Of critical interest is a relatively weak $455 \mathrm{~nm}$ feature associated with a pheophytin anion. As a direct signature of intermediate charge separation, this feature can be used to correlate transient features in the $\mathrm{Q}_{\mathrm{y}}$ band with the charge separation process.

A home-built non-colinear optical parametric amplifier (NOPA) powered by a $1 \mathrm{kHz}$ commercial chirped pulse amplifier (Spectra Physics Spitfire Pro) produces our tunable pump source. The pair of collinear pump pulses with variable relative time delay required for $2 \mathrm{D}$ spectroscopy in the pump probe geometry are created from the NOPA beam using an acousto-optic pulse shaper (Fastlite Dazzler). The pump pulses are also temporally compressed to $30 \mathrm{fs}$ by the pulse shaper. A broadband probe is generated by launching a $800 \mathrm{~nm} 35 \mathrm{fs}$ pulse into a $\mathrm{CaF}_{2}$ crystal, which is kept in constant planar motion to prevent damage to the crystal. To avoid lineshape and kinetic distortions resulting from chirp in the probe we compress the continuum to $30 \mathrm{fs}$ using a pair of extremely broadband chirped mirrors (Layertec) with approximately $-40 \mathrm{fs}^{2} \mathrm{GVD}$ per bounce. Population kinetics are observed by altering the relative delay between pump and probe pulses. The entire detection axis is dispersed onto a cooled CCD (Princeton Pixis) using a 300 groove $/ \mathrm{mm}$ grating (Horiba iHR320) and read out at the laser repetition rate. Noise resulting from probe fluctuations is removed by simultaneously collecting an unpumped reference pulse in the same spectrometer. We employ a four phase cycle detection scheme with probe chopping to remove pump scatter and transient absorption artifacts.

PSII reaction center complexes were prepared from spinach using a slightly modified version of van Leeuwen's protocol [11], where the ratio of peak amplitudes in the Soret band was used as a measure of purity. After elution from the column, samples were centrifuged with membrane filters to remove excess salt and increase concentration. Glycerol was added to the samples in Bis-Tris buffer pH $6.5(67 \% / 33 \% \mathrm{v} / \mathrm{v}$ ) to act as a cryoprotectant. Samples were cooled to $77 \mathrm{~K}$ in a cryostat (Oxford Instruments Microstat N).

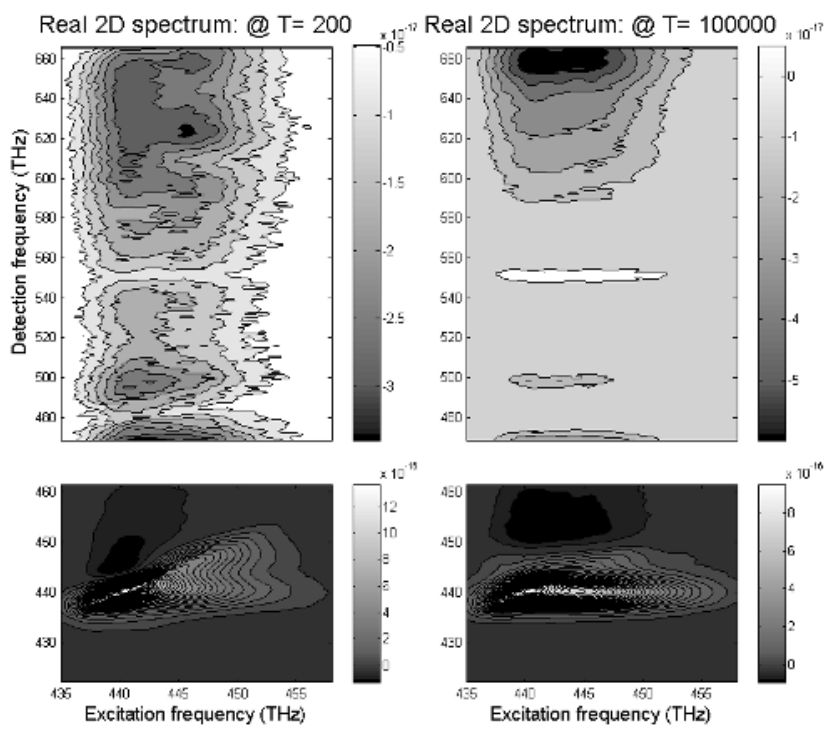

Fig 1: Two dimensional absorptive spectrum of the d1d2 cyt-b559 PSII RC at $77 \mathrm{~K}$ for waiting times of $200 \mathrm{fs}$ (left) and 100 ps (right) in two different frequency regions (top 
and bottom rows). Contours here are on a linear scale (10\% per contour for the top row of figures, and $2.5 \%$ per contour for the bottom row). The signal levels for the top row are as much as 100 times weaker, but features from pheophytin, carotenoids, and chlorophyll are present.

Data was collected over waiting times ranging from 0-180 ps to monitor the appearance and decay of the weak high energy features. Consistent with pump probe studies [9], we find that the $455 \mathrm{~nm}$ (660 $\mathrm{THz}$ ) pheophytin anion signature is appreciable by $\sim 3$ picoseconds. We also observe other transitions of interest, including the chlorophyll a $\mathrm{Q}_{\mathrm{x}}$ and $\mathrm{Q}_{\mathrm{y}}(1,0)$, pheophytin $\mathrm{Q}_{\mathrm{x}}$ and carotenoid bands. In our previous $\mathrm{Q}_{\mathrm{y}}$ studies, we identified the spectral signatures of different kinetic processes using "two dimensional decay associated spectra" (2D DAS). The 2D DAS revealed distinct spectral features on the 1-3 ps timescale that has been associated with the primary charge separation process. The additional information available from the continuum probe studies presented here will enable direct correlations to be made between the spectral changes in the $\mathrm{Q}_{\mathrm{y}}$ band and charge separation events. Also ongoing are efforts to simulate the 2DES data based on current excitonic models [8]. The more extensive data set provided by continuum probe 2DES will allow more rigorous testing and refinement of these models.

\section{References:}

1. M. H. Cho, "Coherent two-dimensional optical spectroscopy," Chemical Reviews 108, 1331-1418 (2008).

2. S. H. Shim, D. B. Strasfeld, Y. L. Ling, and M. T. Zanni, "Automated 2D IR spectroscopy using a mid-IR pulse shaper and application of this technology to the human islet amyloid polypeptide," Proceedings of the National Academy of Sciences of the United States of America 104, 14197-14202 (2007).

3. J. A. Myers, K. L. M. Lewis, P. F. Tekavec, and J. P. Ogilvie, "Two-color two-dimensional Fourier transform electronic spectroscopy with a pulse-shaper," Optics Express 16, 1742017428 (2008).

4. R. E. Blankenship, Molecular mechanisms of photosynthesis (Blackwell Science, Oxford, 2002).

5. S. R. Greenfield and M. R. Wasielewski, "Excitation energy transfer and charge separation in the isolated Photosystem II reaction center," Photosynthesis Research 48, 83-97 (1996).

6. J. P. Dekker and R. Van Grondelle, "Primary charge separation in Photosystem II," Photosynthesis Research 63, 195-208 (2000).

7. T. Renger and E. Schlodder, "Primary Photophysical Processes in Photosystem II: Bridging the Gap between Crystal Structure and Optical Spectra," Chemphyschem 11, 1141-1153 (2010).

8. V. I. Novoderezhkin, J. P. Dekker, and R. van Grondelle, "Mixing of exciton and chargetransfer states in Photosystem II reaction centers: Modeling of Stark spectra with modified Redfield theory," Biophysical Journal 93, 1293-1311 (2007).

9. E. Romero, I. H. M. van Stokkum, V. I. Novoderezhkin, J. P. Dekker, and R. van Grondelle, "Two Different Charge Separation Pathways in Photosystem II," Biochemistry 49, 4300-4307 (2010).

10. J. A. Myers, K. L. M. Lewis, F. D. Fuller, P. F. Tekavec, C. F. Yocum, and J. P. Ogilvie, "Two-dimensional electronic spectroscopy of the D1-D2-cyt b559 photosystem II reaction center complex," Journal of Physical Chemistry Letters 1, 2774-2780 (2010).

11. P. J. van Leeuwen, M. C. Nieveen, E. J. van de Meent, J. P. Dekker, and H. J. van Gorkom, "Rapid and simple isolation of pure photosystem II core reaction center particles from spinach," Photosynth. Res. 28, 149-153 (1991). 